\title{
Microbond multiple fiber pull-out test to evaluate interface properties of UHMWPE/LDPE self-reinforced polymer composites
}

\author{
M Sharan Chandran*, Yashasvi Chebiyyam and K Padmanabhan \\ School of Mechanical Engineering, Vellore Institute of Technology, Vellore, 632014, India \\ *Corresponding Author: sharanchandran.m@vit.ac.in
}

Submitted: $21 / 06 / 2019$

Revised: $19 / 02 / 2020$

Accepted: $02 / 03 / 2020$

\begin{abstract}
Interfacial properties of composite materials play an important role in overall efficiency and reliability of these materials in structural applications. The objective of this study is to develop a multiple fiber microbond pull-out test to determine the interfacial properties of self-reinforced polymer composites (SRPC) and compare it with single fiber multiple fiber pull-out tests. SRPC possess better interfacial adhesion due to their similarity in chemical structure. The system used in this study is LDPE sheet reinforced with plain weave ultra-high molecular weight polyethylene (LDPE/ UHMWPE). The optimal operating temperature was estimated with DSC and TGA analysis. The micromechanical and meso-mechanical approaches were compared to validate the results. A fractographic study was performed to correlate lamina and meso-mechanical properties found in this study. It was observed that the multiple fiber pullout test explained in this study is on par with or better than the other conventional methods to evaluate interface properties.
\end{abstract}

Keywords: Fractography; FTIR; SEM; Interface analysis; Single polymer composites.

\section{INTRODUCTION}

The interface between the matrix and the reinforcement plays major role in load transfer and thus influences the overall efficiency of the composite materials. There are numerous mechanisms by which these interfaces are formed, like adsorption, chemical bonding, physical bonding, mechanical bonding, etc. Interfaces can be characterized by various spectroscopic and optical methods. Fiber fragmentation tests, fiber pull-out test, and fiber push-out test are some of the mechanical testing methods used to evaluate interface properties. Recent experimental studies conducted on interfacial properties of composite materials reveal that the single fiber pull-out is one of the most reliable and simple methods compared to other methods to evaluate the interfacial properties of the polymer composite materials. Understanding the interface properties helps in improving the structural properties of composite materials by enhancing the interfacial adhesion. Some of the disadvantages of single fiber pull-out test are that this method neglects the effect of volume fraction and the requirement of maintaining a critical length of contact between matrix and reinforcement in order to avoid fiber failure before interface de-bonding. These two cases will be addressed in multiple fiber pull-out test compromising with slightly higher scattering of the results. This method can be further improved in the future in order to reduce the scattering (Désarmot and Favre, 1991; Miller, Gaur and Hirt, 1991). 
Interfacial shear stress is the important parameter to be determined in microbond pull-out tests. This property is evaluated by considering the debonding force when the interface crack is initiated under loading. There will be a shearlag region after this event, which cannot be reliably measured. Serge et al. propose a new method for evaluating IFSS without considering the debonding force, which helps in improving the accuracy in evaluation of IFSS (Zhandarov and Mäder, 2016).

Single fiber microbond pullout test for polymer composite materials was explained and established in detail by Wang, Muri, Millert and Gaur, which is the most adapted reference for this study (Wang et al., 2013; Minnicino and Santare, 2012; Jiang and Penn, 1992). A detailed review by Gao C regarding development of self-reinforced composites using different methods and the importance of each was discussed; several of these methods have been employed to study the new composites being developed (Gao et al., 2012). Following the research by I.M. Ward and P.J. Hine in development and testing of various Polyethylene (PE) single polymer composites, it has become vital to develop and study new PE based composites(Morgan et al., 2009; Bárány, Izer and Karger-Kocsis, 2009).

The study aims to understand static properties of the developed self-reinforced composite with approach from various levels. This includes micromechanical, meso-mechanical, and macromechanical approaches. The study provides strong arguments by comparing traditional methods with experimental work performed. The results are critically compiled, which shows the importance of various approaches for static study. In the process, they are validated in terms of ease of use and accuracy likewise.

For this purpose, this study is conducted using standard quality polymers, LDPE sheet $\left(\sim 120 \mu \mathrm{m}, 0.92 \mathrm{~g} / \mathrm{cm}^{3}\right)$ obtained from New Pilot Plastics, Chennai, and UHMWPE plain weave fabric $\left(0.95 \mathrm{~g} / \mathrm{cm}^{3}\right)$ shipped by Chinese manufacturer, Shenzhen Bofeng International Logis. The microbond pullout method, developed by Padmanabhan $\mathrm{K}$ (Krishnan, 2017) is used with modifications and refinement to determine the in plane shear modulus. The results of macro-, micro-, and meso-properties so obtained are reported in the results section. Authors have also conducted similar analysis in different systems of SRPCs like High Density Polyethylene (HDPE-PET/HDPE) and Polypropylene (iPP/aPP) systems (Sharan and Padmanabhan K, 2019; Sharan et al., 2019). Authors have also studied the static and dynamic mechanical propeorties of PE and PP self reinforced composites and established the correlation between static and mechanical properties of these composites with their interface properties(Chandran and Padmanabhan, 2020; Sharan Chandran et al., 2014).

A comparative analysis of single fiber and multiple fiber pull-out tests is carried out in this study. The analysis is carried out in single, double, and triple fiber bundle systems. It helps in understanding the variation of interface properties with change in number of fiber bundles. Each extracted fiber from the fabric is a bundle of number small fibers. Extracting individual small fibers from the extracted fiber bundle makes the sample preparation complex, and the possibility of fiber breakage before the interface failure increases. Thus, each fiber will be treated as a fiber bundle in this study. Figure 1 is a schematic representation of a microvise, which is used to hold the blob/fiber bundle assembly in testing. Details of materials used in the study and their properties are listed in Table 1.

Numerous efforts to increase the interface properties have been reported recently. Chao Zhi et al. related the Interfacial shear strength (IFSS) and other interface properties of fiber micro-balloon epoxy ternary composites gained through experimental method of Microbond test and with numerical results. They described that the fiber diameter, volume fraction, and size of the micro-balloon particle have significant impact on IFSS (Zhi et al., 2018). Yunyun Ma et al. (2018) studied the IFSS estimated by microbond test of graphene oxide grafted carbon fiber, which increases IFSS significantly. Mechanical properties of thermosetting polymer composites are also dependant on their interface characteristics. Engineered interfaces through various fibre surface treatments will improve the mechanical properties by developing a modified interface.(Raman et al., 2013; Raman, Salil Kumar and Sharan Chandran, 2014; Chandran et al., 2018) 


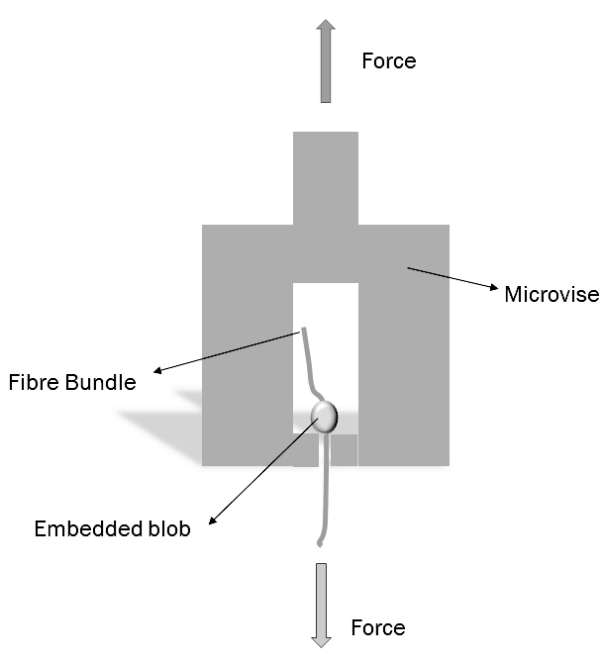

(a)

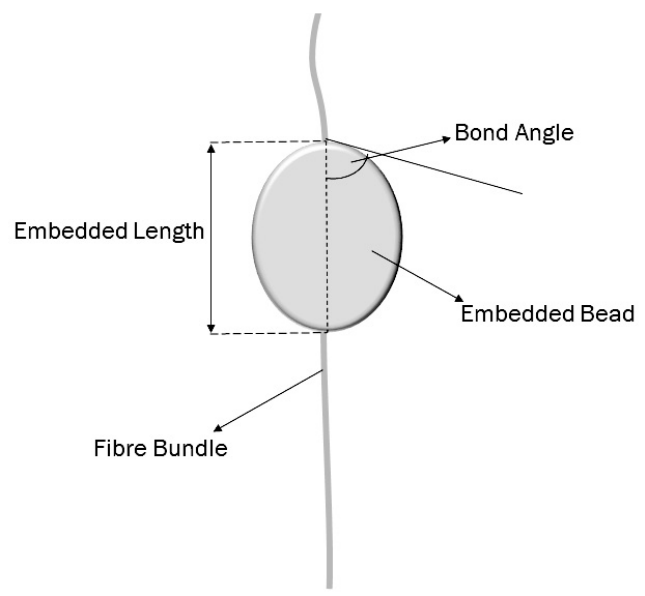

(b)

Fig. 1. (a) Schematic diagram of pull-out (b) blob-fiber assembly.

Table 1. Properties of the components.

\begin{tabular}{|ll|}
\hline \multicolumn{2}{|c|}{ Matrix: LDPE } \\
\hline Density: & $0.92 \mathrm{~g} / \mathrm{cc}$ \\
\hline Melting temperature: & $125^{\circ} \mathrm{C}$ \\
\hline
\end{tabular}

\begin{tabular}{|c|c|}
\hline \multicolumn{2}{|c|}{ Apparent matrix viscosity $\left(190^{\circ} \mathrm{C}\right)$} \\
\hline (a) shear Rate $1000 \mathrm{~s}^{-1}$ : & $265 \mathrm{Ns} / \mathrm{m}^{2}$ \\
\hline \multicolumn{2}{|c|}{ Fabric: UHMWPE } \\
\hline Density & $0.93 \mathrm{~g} / \mathrm{cc}$ \\
\hline Melting point: & $145^{\circ} \mathrm{C}$ \\
\hline Time of heating & 45 minutes \\
\hline Fiber surface: & untreated \\
\hline Strain rate: & $2 \mathrm{~mm} / \mathrm{min}$ \\
\hline
\end{tabular}

\section{MICROMECHANICAL APPROACH}

The micromechanical approach followed in this study is in respect with Chamis and Rosen (E,J Barbero,1999) semi-empirical models. The interfacial properties were evaluated mainly by using elastic modulus of fiber and matrix, volume fraction of fiber and matrix, blob contact length, and average bundle diameter. The Chamis formula for in plane shear stress, $\mathrm{F}$, is given in

$$
F=\left[1+\left(V_{f}-\sqrt{V_{f}}\right) *\left(1-\left(G_{m} / G_{f}\right)\right] * \tau_{m u}\right.
$$

where 
$V_{f}$ is the volume fraction of fibers, and $G_{m}$ and $G_{f}$ are the shear moduli of matrix and fiber, respectively. $\tau_{m u}$ is the ultimate tensile strength of the matrix, while $\mathrm{V}_{\mathrm{f}}$ is known and can vary from 0.2 to 0.8 in practical sense. The values of Elastic modulus of matrix and fiber along with $\mathrm{V}_{\mathrm{f}}$ and $\tau_{m u}$ are input data for CADEC software. This gives an output for $\mathrm{G}_{\mathrm{m}}$ and $\mathrm{G}_{\mathrm{f}}$ in $\mathrm{MPa}$. According to Rosen's prediction, shear stress is modified, as in

$$
\tau_{c o m p}=F * \tanh \left(\gamma * G_{12} / F\right)
$$

where $\tau_{\text {comp }}$ is the modified shear stress prediction, $\gamma$ is the shear strain at failure, and $\mathrm{G}_{12}$ is the shear modulus of the composite. Again, $\mathrm{G}_{12}$ is obtained from CADEC software in the same window as discussed above. Both Chamis and Rosen formulas predict the shear strength of the composite with similar accuracy due to common parameters being used. In this study, the meso-mechanical approach is experimentally applied to compare results with other approaches. This involves the use of microbond pullout test to calculate the interfacial properties with good accuracy. This method is preferred due to ease of formulation and evaluation of required interfacial properties. The intrinsic bond strength, $\tau$, is calculated from

$$
\tau=\mathrm{F} /(\pi . \varnothing . \mathrm{L})
$$

where $\mathrm{F}$ is the difference between peak force needed to debond the matrix blob from the fiber bundle and the frictional force. In this case, a microvise was used, which eliminates any frictional trace. The average diameter of fiber bundle and the blob contact length are represented by $\varnothing$ and L, respectively. As it is already known that the matrix is weaker than the fiber material, the intrinsic bond strength becomes peak debonding force divided by the actual outer interfacial area. Although this condition would not hold for small volume fractions with large fiber bundle diameter, the materials concerned here fall well in the conditions established. The contact angle ( $\theta$ ) between matrix blob and fiber bundle produces a force, which compresses the matrix onto the fiber bundle. This force acts against the shearing process during the pullout test. Thus, this force $\mathrm{F}_{\mathrm{c},}$ is equal to $\mathrm{F} \cot \theta$. Here, $\mathrm{F}$ is the direct force that accounts for the compressive component of the force.

$$
\mathrm{F}=\mathrm{F}_{\mathrm{ad}}+\mu \cdot \mathrm{F}_{\mathrm{c}}
$$

where

$$
\mathrm{F}_{\mathrm{ad}}=\mathrm{F}_{\max }=\tau \cdot \mathrm{A}
$$

Again, $\mathrm{A}$ is the considered interfacial cylindrical area of the interface. The $\mathrm{F}_{\max }$ is the maximum force, which is also the debonding force required. $\mu$ is the coefficient of friction as the fiber bundle is being pulled out at $2 \mathrm{~mm} / \mathrm{min}$ strain rate.

$$
\mathrm{F}=\tau \cdot \mathrm{A}+\mu \cdot \mathrm{F}_{\mathrm{f}} \cot \theta
$$

Unlike the peak debonding force, which is a specific value at any given time of the test, $\mathrm{F}_{\mathrm{f}}$ is the variable force downward as observed in the load-deflection plot. It is important to note that the value of coefficient of friction likely changes between frictional sliding, frictional onset (see Equation (4)), and $\mathrm{F}_{\max }$. Hence, dividing Equation (6) by A gives frictional stress value. This is manifested and interpreted as the interfacial shear stress (IFSS) also.

$$
\mathrm{F} / \mathrm{A}=\mathrm{IFSS}=\tau+\left(\mu \tau_{\mathrm{f}} \cot \theta\right)
$$




\section{THERMAL CHARACTERIZATION}

Differential scanning calorimetry (DSC) is an essential tool to recognize various stages of thermal processing of polymers as a function of heat flow. Changes in heat flow indicate glass transition, crystallization, and melting points of the polymer. Thermogravimetric analysis (TGA) is a method to record various stages of thermal degradation. Using these two analyses, proper processing window for thermal processing of the laminate, as well as the microbond, is decided. Upon analyzing the DSC curves for LDPE and UHMWPE, the range of possible processing temperature was identified. As the method employed is hot compaction, identifying this optimum compaction temperature is essential. Under the heat treatment process, the matrix is expected to melt, while the reinforcing fibers are expected to be intact.

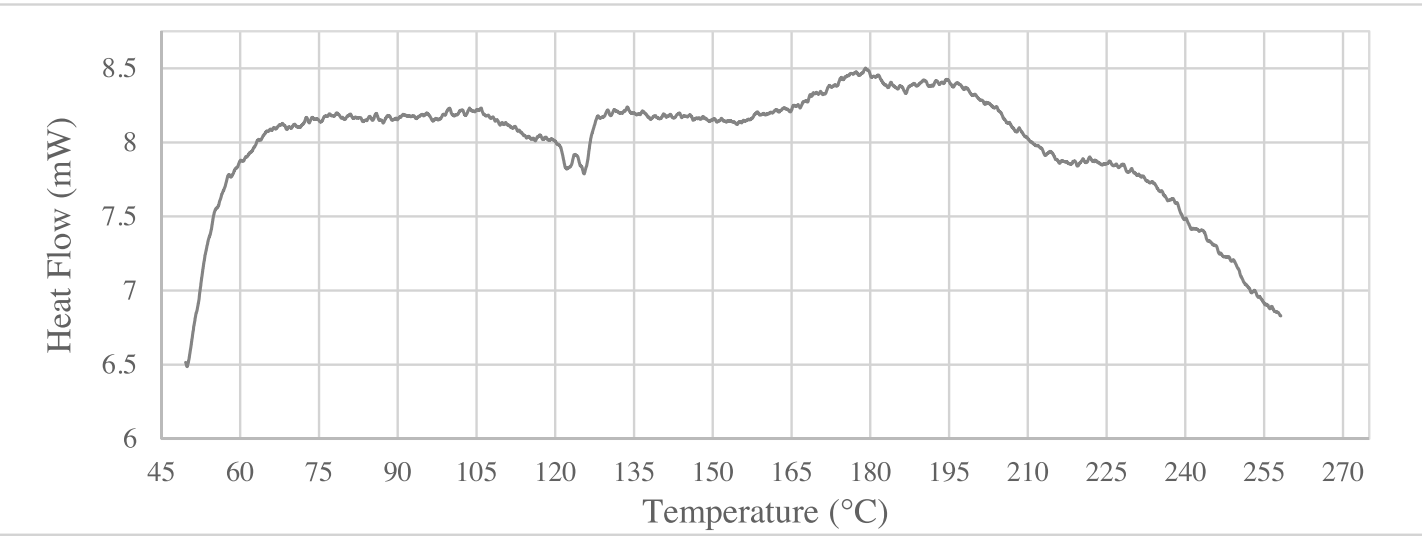

(a)

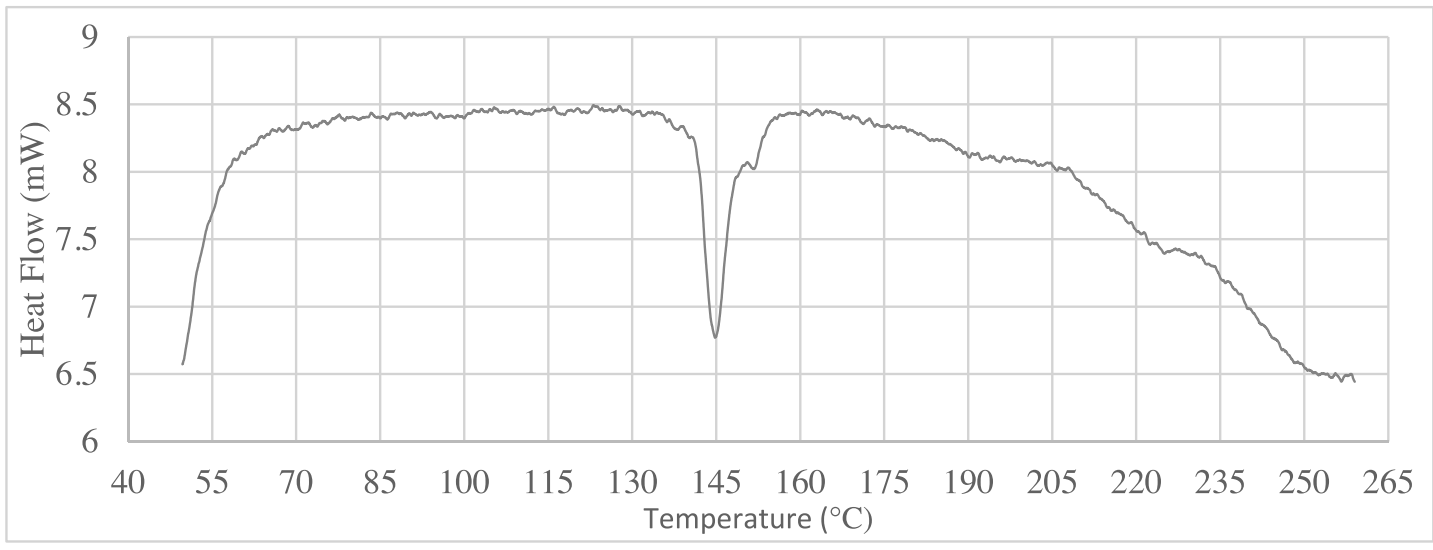

(b)

Fig. 2. (a) DSC curve for LDPE and (b) DSC curve for UHMWPE.

As observed from Figure 2, the processing window is identified as a range of temperatures, where the LDPE melts partially, but UHMWPE does not change phase. This happens between $125^{\circ} \mathrm{C}$ and $140^{\circ} \mathrm{C}$. The processing temperature is fixed after trial and error. The LDPE partially melts only to recrystallize forming bond/adhesion with the fiber bundle, which happens best at $136^{\circ} \mathrm{C}$. Thermogravimetric analysis chart in Figure 3 explains thermal stability of the materials within the processing window. 


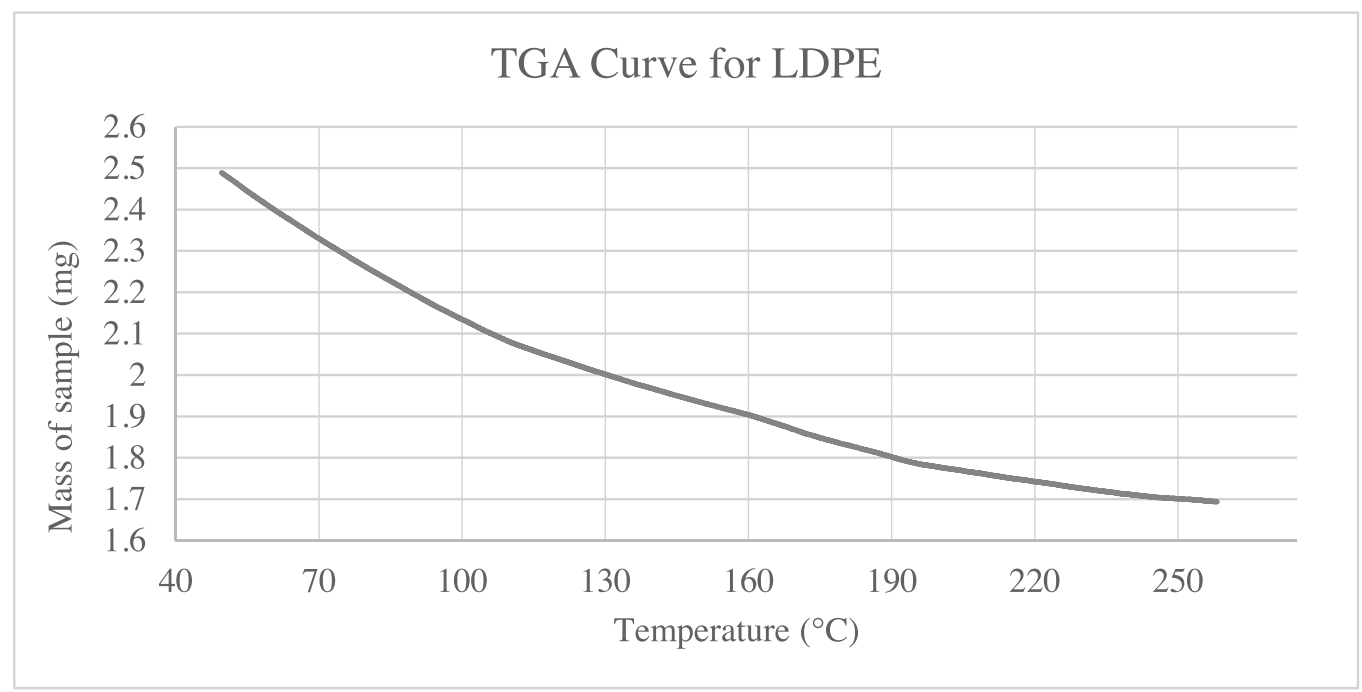

(a)

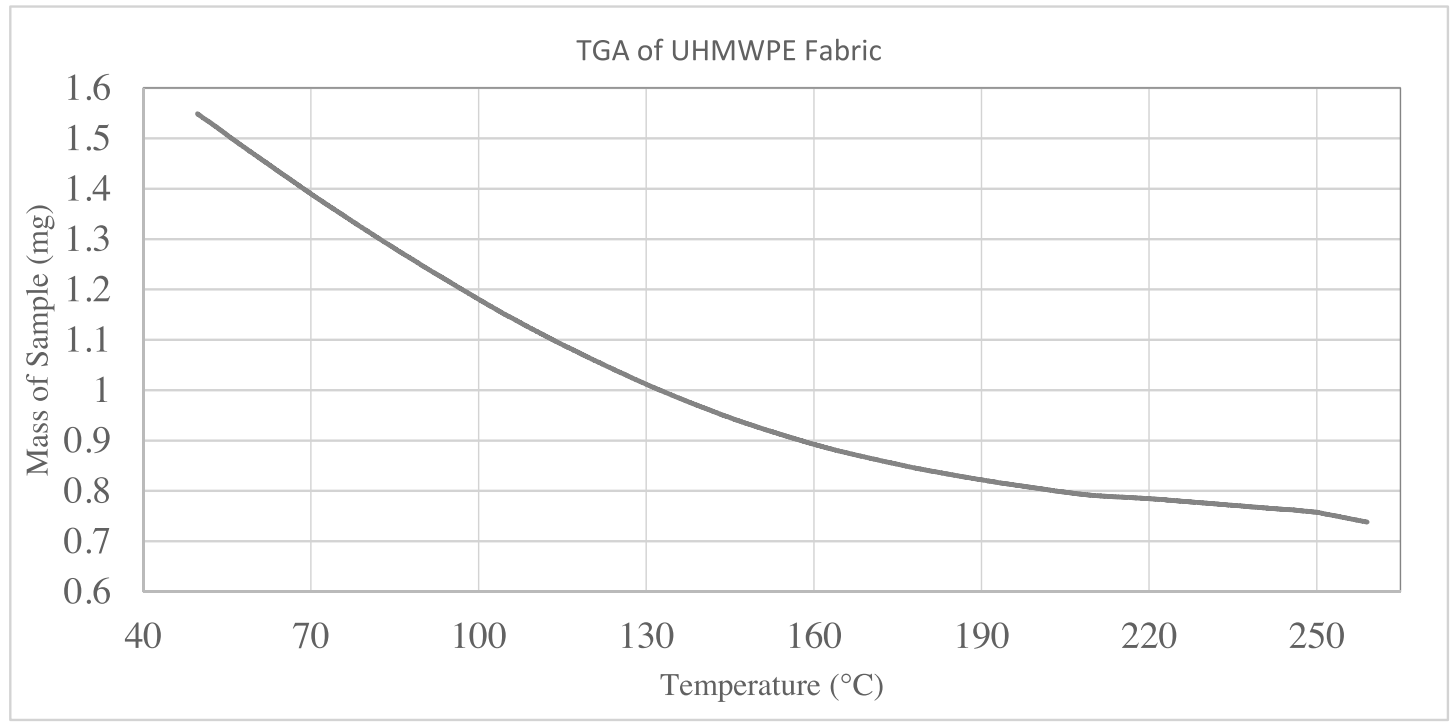

(b)

Fig. 3. (a) TGA curve for LDPE (b) TGA curve for UHMWPE.

\section{SAMPLE PREPARATION FOR PULL-OUT TEST}

For sample preparation, square size LDPE of $5 \times 5 \mathrm{~mm}$ was cut firstly. Then, four of such pieces were used for one sample preparation. Each piece was pierced through the center to allow the fiber bundle to pass. For the fiber bundle preparation, individual fiber bundles from the fabric were pulled out carefully. Two such fiber bundles were then joined to form two-fiber bundle $\left(\mathrm{V}_{\mathrm{f}}=0.6\right)$. Similarly, three fiber bundles pulled out from fabric were joined to create threefiber bundle $\left(\mathrm{V}_{\mathrm{f}}=0.7\right)$. These multi-fiber bundles were then passed through four matrix pieces each. The processing was done at $136^{\circ} \mathrm{C}$ for about 90 minutes. The samples were cooled in the oven and collected for pullout testing after 10 hours. Figure 4 shows the processed two-fiber and three-fiber bundles. The Prepared samples were taken for the microscopy to measure various parameters like blob size, fiber bundle size, contact angle, etc. (figure 5). 


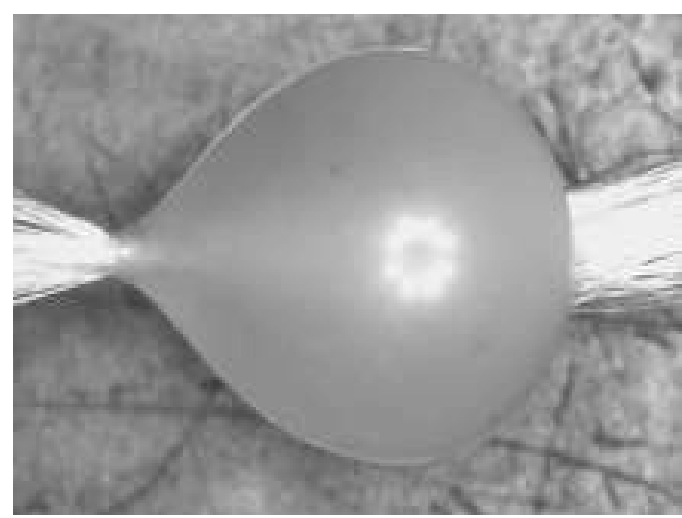

(a)

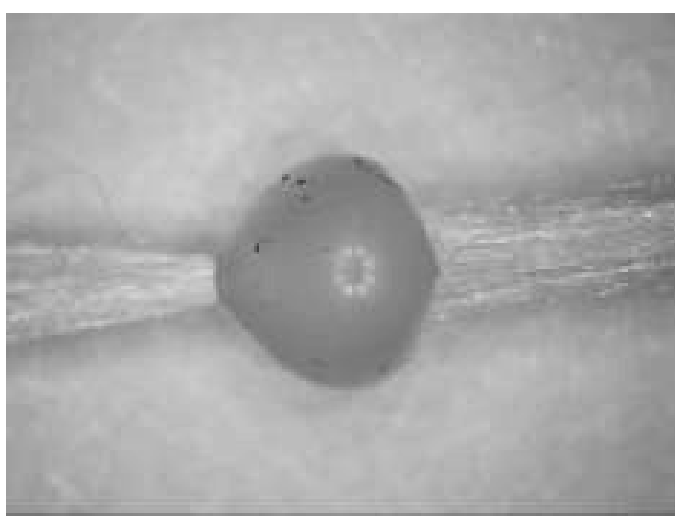

(b)

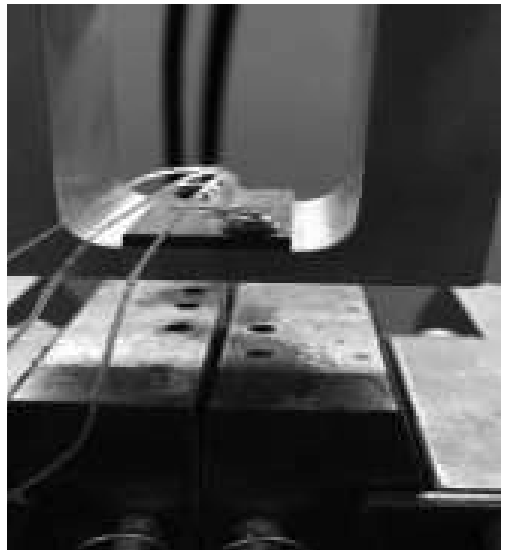

(c)

Fig. 4. Magnified images of processed (a) two-fiber pullout sample and (b) three-fiber pullout sample (c) assembled in microvise.
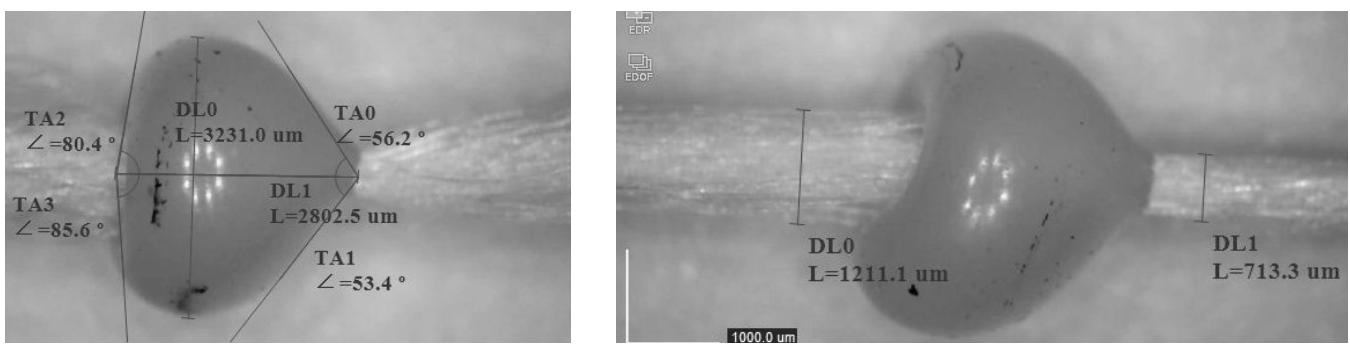

Fig. 5. Magnified image of processed (a) two-fiber pullout sample and (b) three-fiber pullout sample.

\section{FOURIER TRANSFORM INFRARED SPECTROSCOPY (FTIR)}

The FTIR test is often used to classify materials as polymeric, organic, or inorganic. Here, FTIR analysis was conducted on equipment of Thermo Fisher Scientific, and the mode of analysis is KBr Disc method. The basic principle is that when the material is exposed to radiation of various wavenumbers, it tends to absorb only certain radiation and convert it to intermolecular vibration. Thus, the plot of percentage transmittance of radiation from the material versus the wavenumber gives a unique spectral fingerprint. This can be used to characterize material, as well as to check for adhesion. 


\section{SEM ANALYSIS}

ZEISS EVO 18 scanning microscope is used for the fractography with a coupled assistance from smartSEM software. Electron beam generated from Lanthanum hexaboride $\left(\mathrm{LaB}_{6}\right)$ gun passed through gold coated samples of PE and PP. With an accelerating voltage of $5 \mathrm{kV}$, various fracture features could be studied from the micrographs. Extensive fractography is carried out on failed sample of all the meso-mechanical, quasi-static, and drop mass impact tests.

\section{RESULTS AND DISCUSSION}

\section{Specimen 7 to 7}

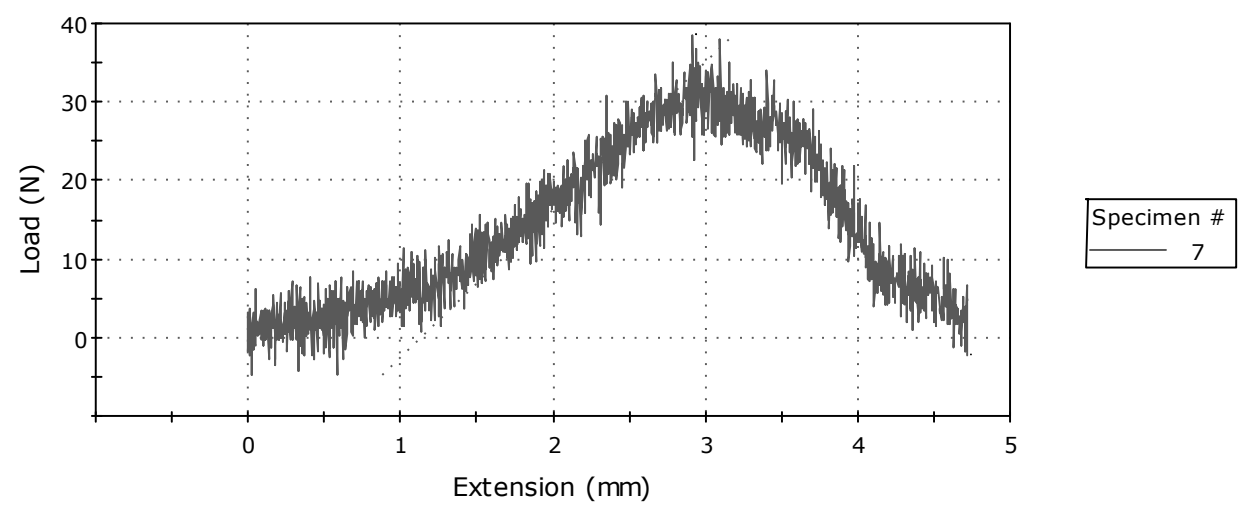

Fig. 6. Load Vs Extension plot.

The meso-mechanical approach is a good mix of calculations and experimentation. Table 2 shows the measured parameters and IFSS of single, double, and triple fiber bundles pull-out samples.

Load-deflection plot (Figure 6) usually has three distinct features. They are the point of debonding, point of maximum load, and pure frictional slide region. In this study, debonding point was not well noticeable in the curve. In sample figure 6, maximum load point can be noticed, and the blob slides immediately after that. This sliding is controlled and resisted by frictional force between the blob and the fiber bundle, which can be visualized as a comparatively lower slope region immediately after attaining the maximum load.

Contact angle of the matrix with the fiber is an indication of its wettability and overall interface properties. It can be observed that the contact angle gradually increases slightly when the blob length reduces. Contact angle is less for single fiber bundle and slightly higher for double and triple bundles. Blob length is reducing when the bundle size is increasing. This indicates that the there is a requirement to maintain a critical blob length, as well as critical blob angle in order to get an optimum result. Wettability and, thus, the interface properties are highly dependent on these parameters. Higher contact angle is an indication of lower wettability.

The distinction between two-fiber bundle and three-fiber bundle is clearly noted from the average IFSS values from both samples. The statistical nature of this approach often requires averaged values. In this case, the average IFSS is $4.23 \mathrm{MPa}$ and can be used for all practical purposes. From Table 2, we can see that the contact angles fall in the range of 47-77 degrees, while the IFSS ranged between 1.8 and 10.4MPa. For a small change in volume fraction, contact angle, or area of contact, the deviation in IFSS is significant. This again shows the statistical nature of the pull-out test. 


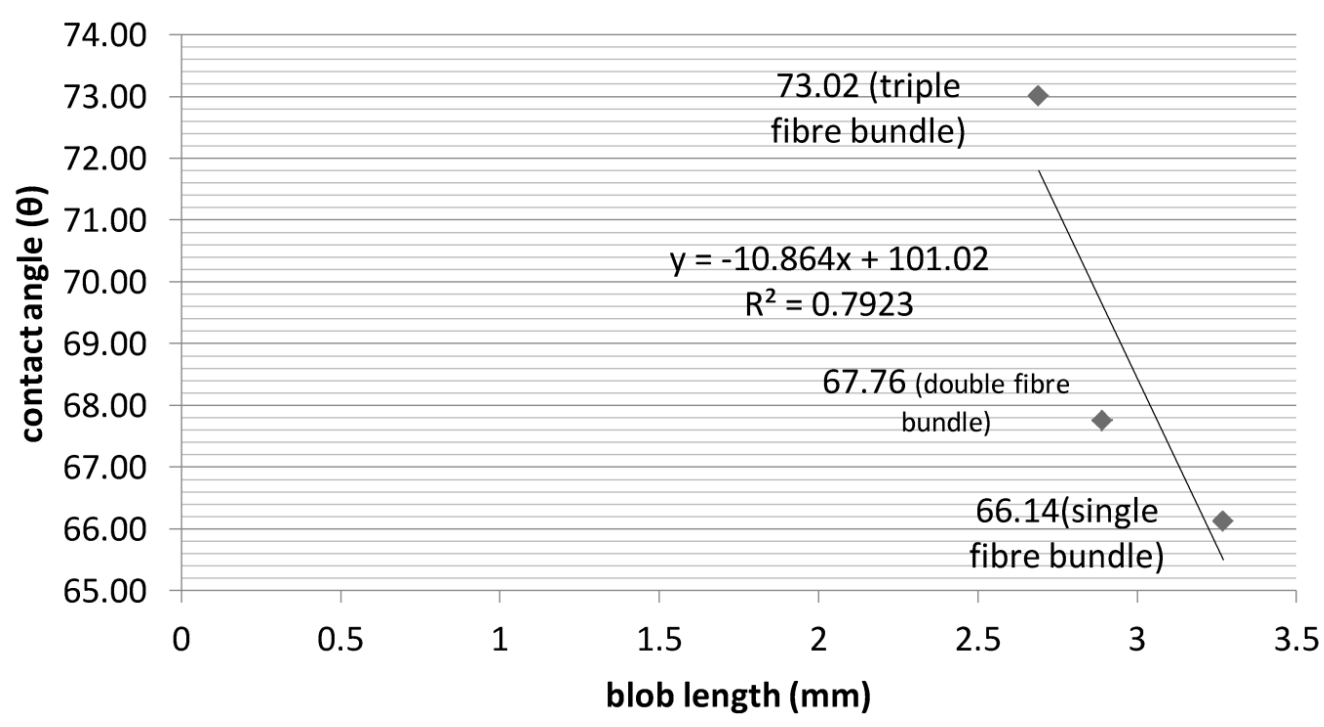

Fig. 7. blob length versus contact angle.

Peak pull-out force can be seen more dispersed when the bundle size increases (figure 7). When the bundle size increases, the possibility of internal crack also increases. Bundle may also lead to the occurrence of voids in the interface. This may be the reason increased dispersion of the data. The frictional resistance has more converged data with single fiber bundle compared to multiple fiber bundles. Double fiber bundle data is more dispersed, while the three fiber bundle data seems to be with less dispersion. This again indicates the necessity of deciding an optimum bundle size in order to get better results. Friction is expected to increase when the surfaces of contact increase, but the existence of internal non-contact region will be more when the bundle size increases.

Interfacial shear stress is one of the most important parameters to be evaluated in interface properties. It can be seen that the blob length formation is very much consistent in single fiber compared to double fiber bundle and triple fiber, and a reduction in IFSS with increasing blob length is notable. This indicates the presence of some other factors influencing the interface strength other than the contact surface. When the blob contact angle increases for single and double bundles, IFSS has almost a gradually increasing trend. But when the blob angle becomes obtuse, this phenomenon will reverse because of lower wettability. But for triple fiber bundle, higher blob contact angle leads to a considerable reduction in IFSS, which is due to the reduction in adhesion and wettability. All these indicate the necessity of maintaining an optimum contact angle and blob length. From the initial discussion, it can be observed that these two parameters are interrelated and can be maintained in close observation, while the samples are prepared.

Figures 8-12 are the plots of influence of evaluated interface properties with those of bond length and contact angle. As these parameters are difficult to control, the influence of these parameters was very much significant in the test. Higher dispersion (low $\mathrm{R}^{2}$ ) in data indicates lower influence of bond length/contact angle on these interface properties. Thus, it gives more freedom in fabrication of the samples. 


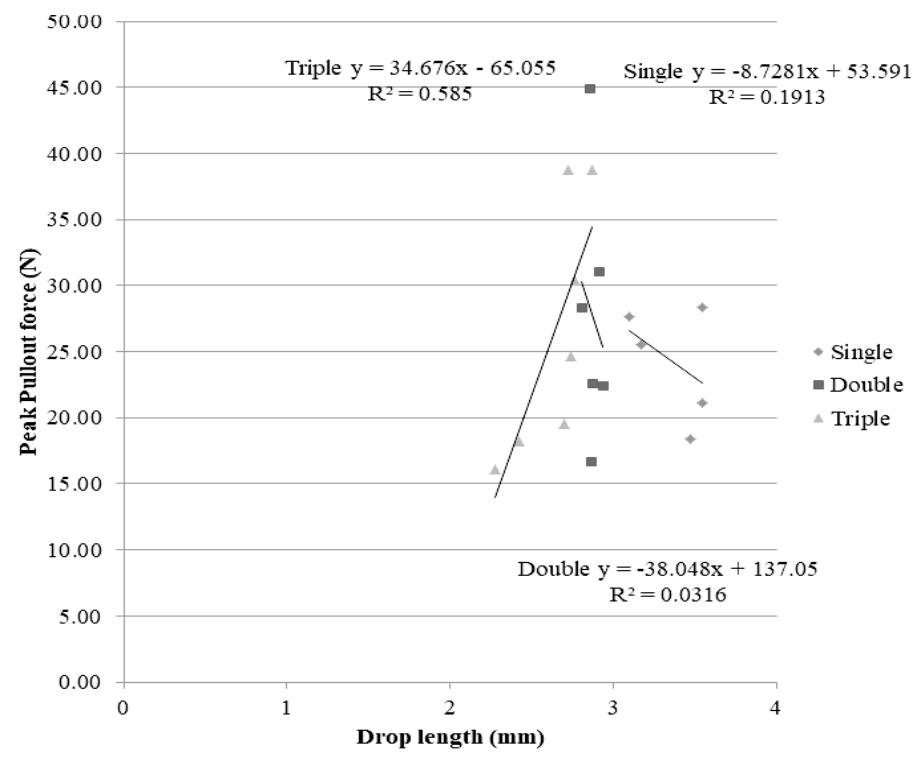

Fig. 8. Peak pullout force Vs blob length (mm).

The trend line shows statistically that as the blob length increases, the maximum force required to debond the interface also increases (Figure 8). This means that a smaller size blob will tend to shear off for a smaller pullout force. Within the selected range, drop length has least influence on peak pullout force, and the peak pullout force is highly influenced by drop length in triple bundle pullout.

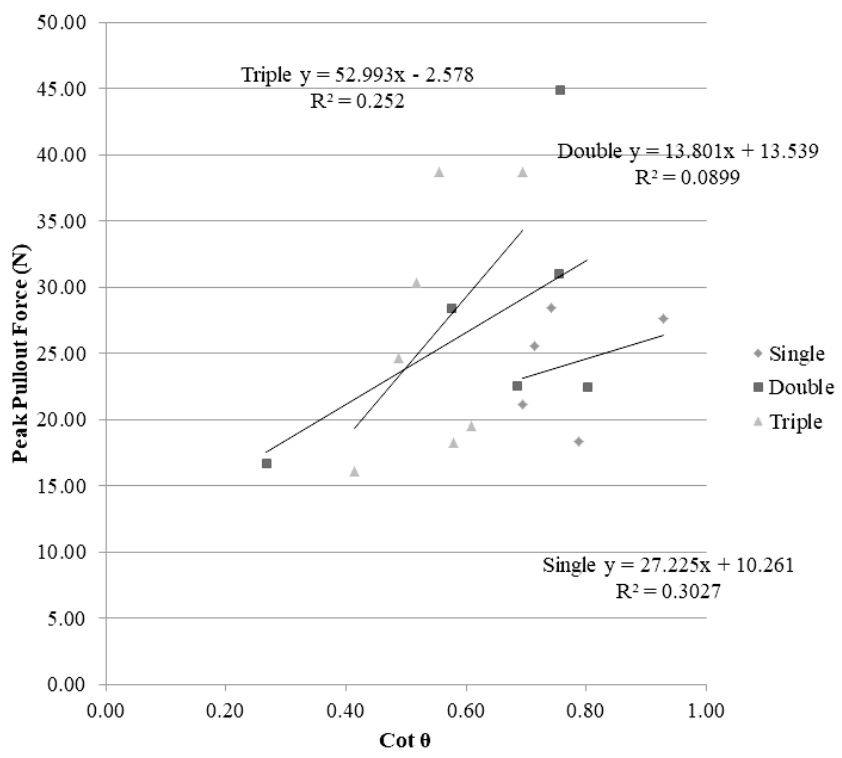

Fig. 9. Peak pull-out force Vs contact angle. 


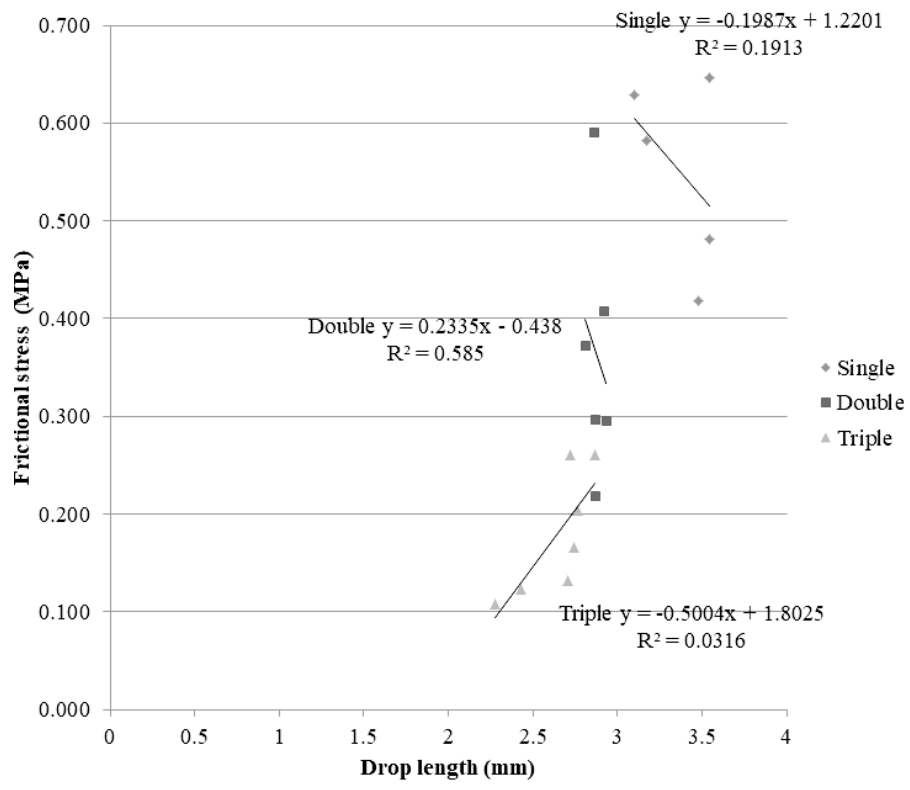

Fig. 10. Frictional stress Vs blob length plot.

Increasing contact angle to obtuse angle reduces the wettability thus reducing the interface strength in single fiber pullout, but not within the range of acute angle as observed in plot and influenced by other parameters in multiple fiber pullout (Figure 9). There is also a correlation between the IFSS, blob length, and contact angle. Due to the over dispersion, double bundle provides an unrealistic trend for frictional stress also (Figure 10). This can be stated with the plots of IFSS, showing that as blob length increases, the IFSS shows an increase too (Figure 11). This can partly be attributed to the fact that as the blob length increases, the interface 'stretches' along the axis of fiber bundle. This causes the contact angle to decrease. As a result, the samples with more acute angles of contact had higher IFSS (Figure 12). Increase in contact angle has more influence in triple fiber bundles.

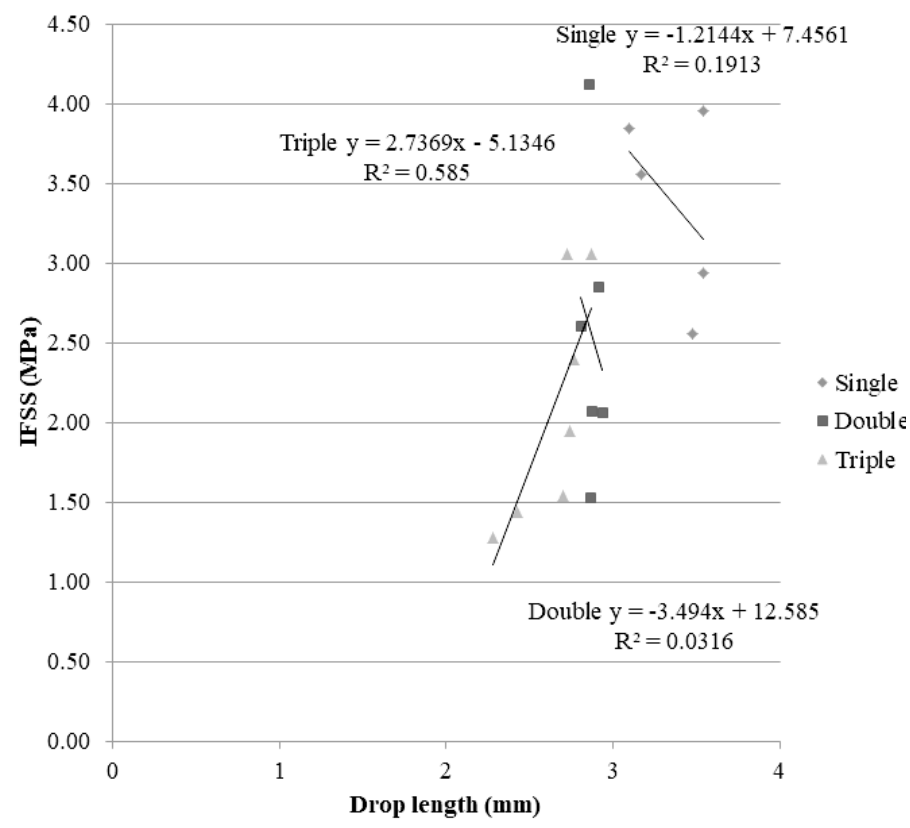

Fig. 11. IFSS Vs blob length plot. 


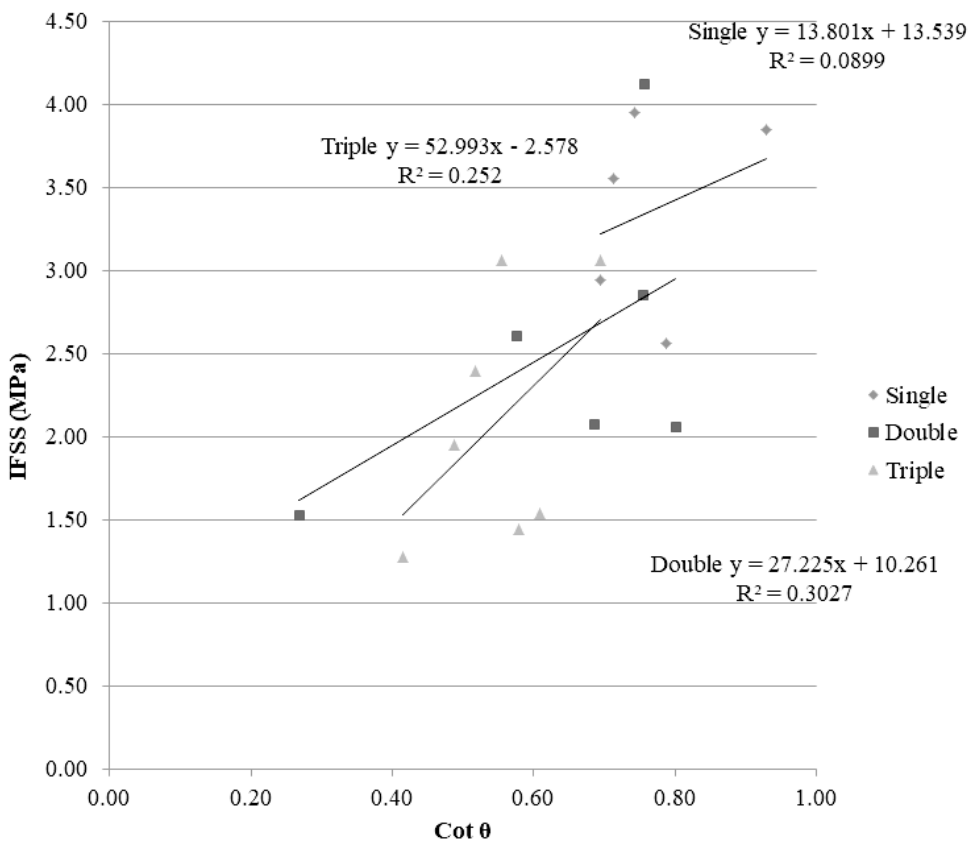

Fig. 12. IFSS Vs blob contact angle.

The plot of IFSS versus fiber bundle diameter in Figure 8 also shows a similar correlation to that of IFSS vs contact angle. As the bundle diameter increases, more fibers are present at the same cylindrical interface. This means that a lesser pullout force is needed to shear the interface due to poorer adhesion of each fiber with the matrix blob. This is clearly shown by decreasing IFSS values.

\section{MICROMECAHNICAL COMPARSION}

The micromechanical approach adopted by Chamis and Rosen formulations predict the interfacial properties like shear stress (IFSS). The IFSS versus volume fraction of fibers plot in Figure 13 compares this result with other approaches.

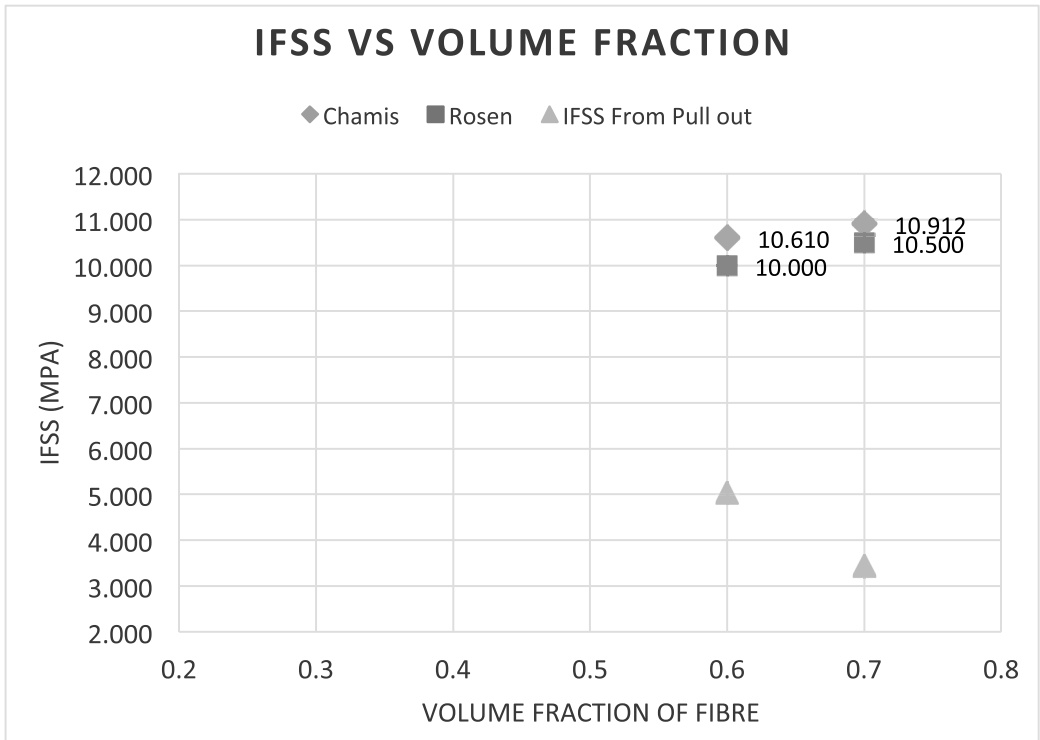

Fig. 13. IFSS vs Volume fraction plot for macro-, meso- and micromechanical approaches. 
Micromechanical approach is conservative in the sense that it fails to consider the effect of wettability at the interface of the fiber bundle and the blob. Since the actual properties of the material are obtained by macromechanical experimentation, it is safe to set this result as reference for all arguments. So, the observed deviation of IFSS obtained by micromechanical approach is more than that obtained by meso-mechanical approach (pull-out testing). Thus, mesomechanical values are more accurate in predicting properties of the bulk of the material.

\section{FTIR COMPARISON}

Figure 14 shows the result for FTIR analysis of the constituent polymers of the composite developed. At 2850 $3000 \mathrm{~cm}^{-1}$, the C-H bonds experience strong asymmetrical and symmetrical stretching. The peak at $1467 \mathrm{~cm}^{-1}$ shows bending deformation of $\mathrm{CH} 2$ and $\mathrm{CH} 3$ bonds at medium intensity. The peak at $717 \mathrm{~cm}^{-1}$ shows a weak $\mathrm{CH} 2$ rocking bend deformation. Peaks between 2850 and $3000 \mathrm{~cm}^{-1}$ show a strong $\mathrm{CH} 3, \mathrm{CH} 2$, and $\mathrm{CH}$ bond stretching. Peak at $1467 \mathrm{~cm}^{-1}$ shows bending deformation of $\mathrm{CH} 2$ and $\mathrm{CH} 3$ bonds with medium intensity. Peak at $715 \mathrm{~cm}^{-1}$ shows weak $\mathrm{CH} 2$ rocking type bending. There are not much noticeable changes before and after the pullout except some regions indicating the adhesion.

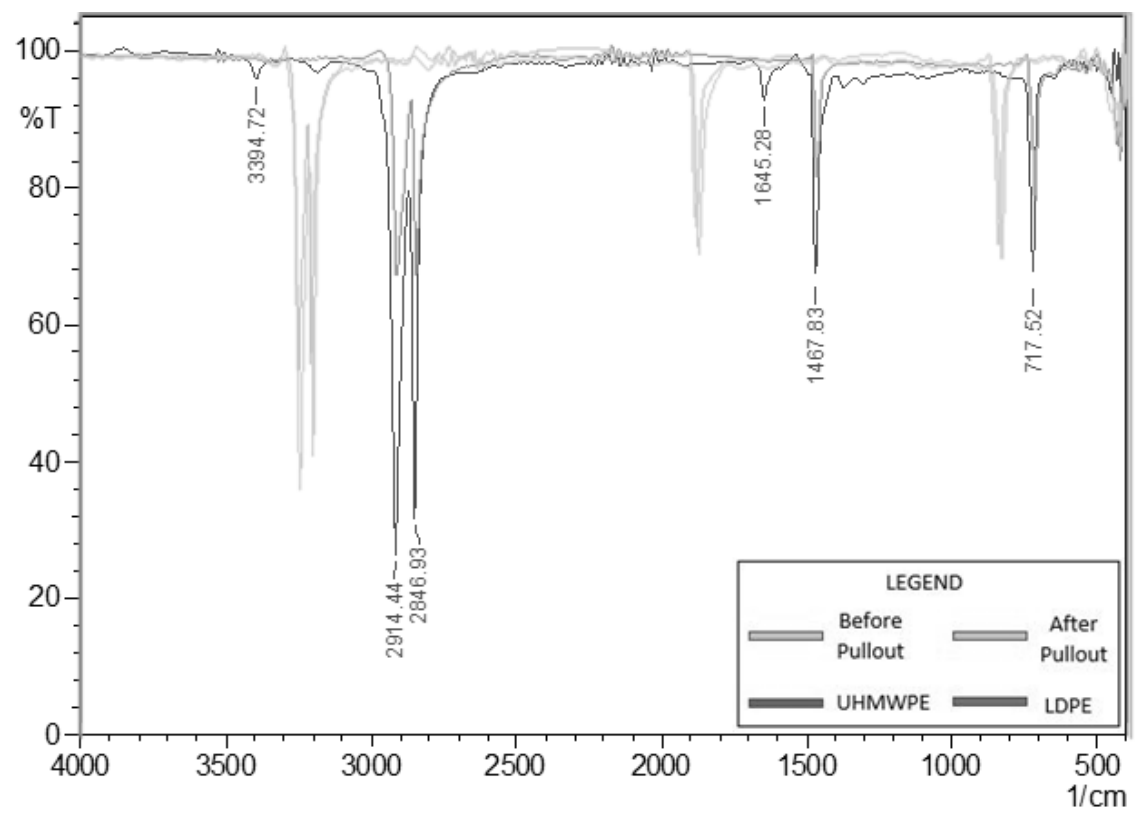

Fig. 14. FTIR comparison chart.

\section{FRACTOGRAPHY OF PULL-OUT SAMPLES}

As the pullout test was performed, few samples did not suffer shear failure and rather failed due to tensile forces. In some cases, the blob was not attached to the fiber bundle in a satisfactory way, which caused abnormally low peak pullout force for that sample and was eventually eliminated from calculations. The samples observed to undergo shear failure were viewed clearly under scanning electron microscope (SEM). It was performed using Carl Zeiss Optics of the equipment, which could magnify the samples from $30 \mathrm{x}$ to $800 \mathrm{x}$ for clear view and images. 


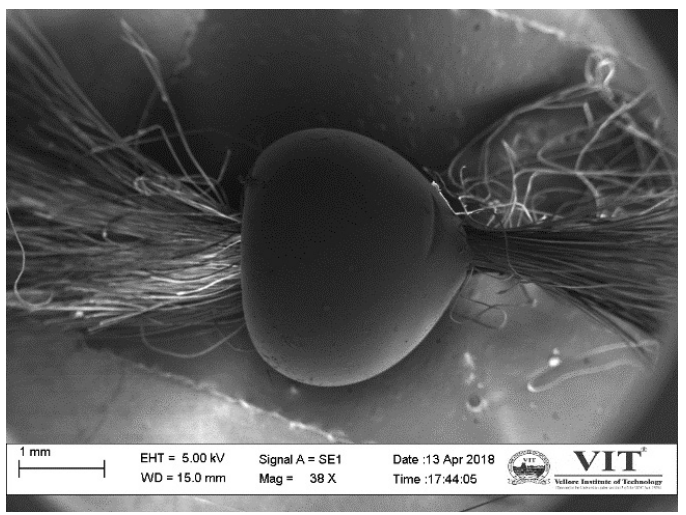

(a)

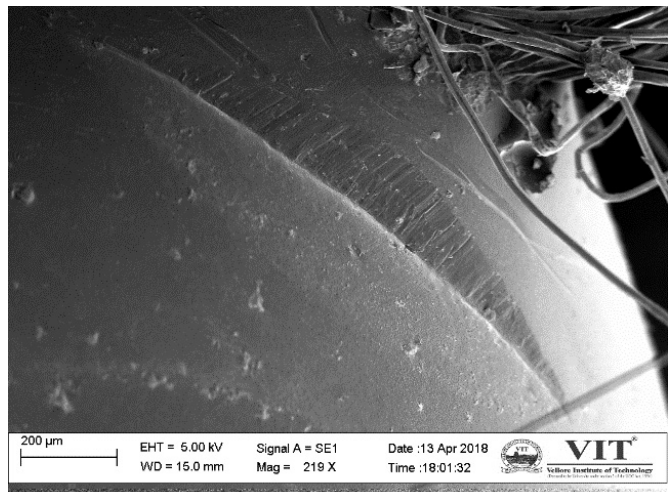

(c)

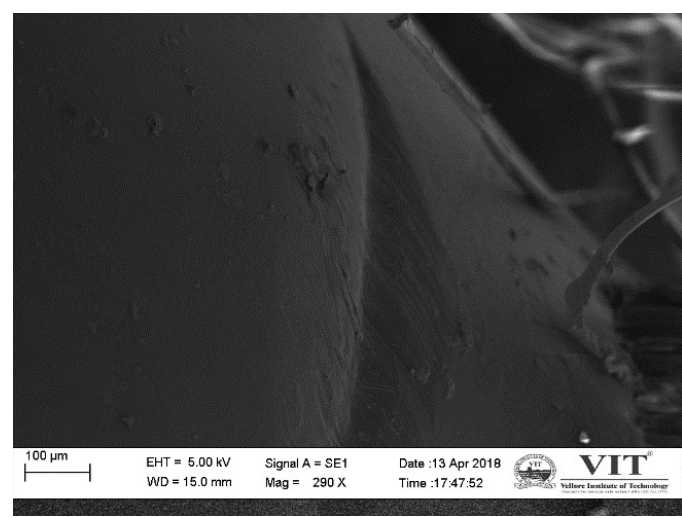

(b)

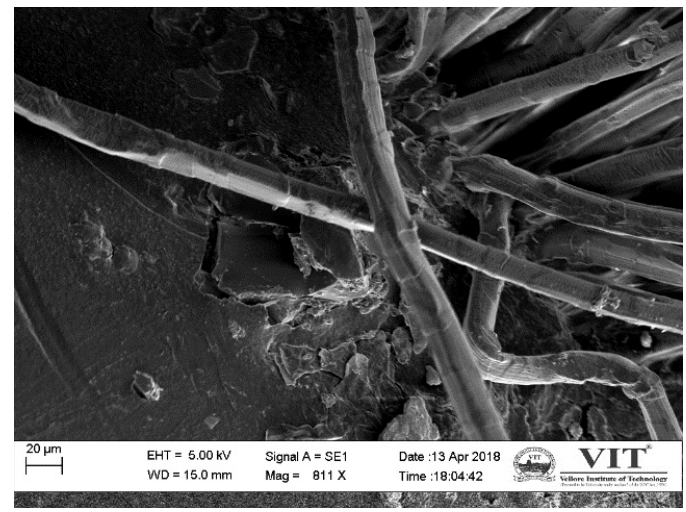

(d)

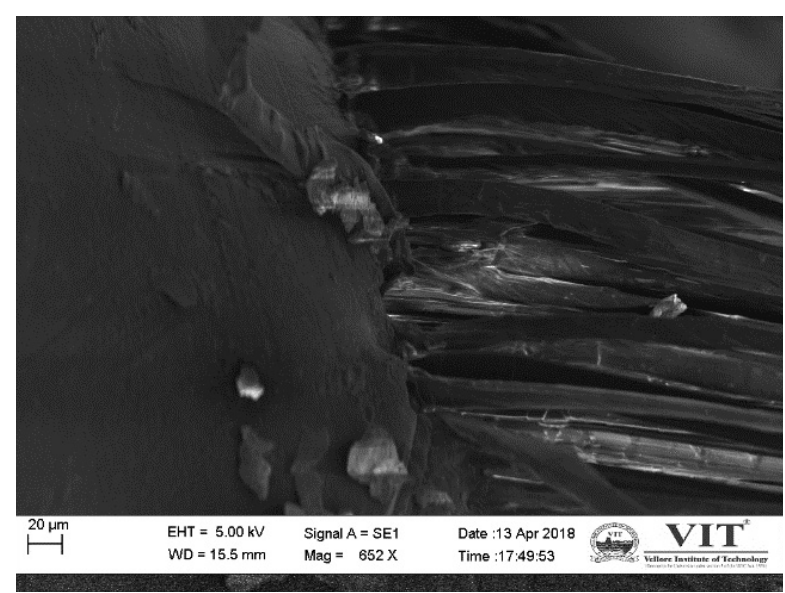

(e)

Fig. 15. Fractography.

Figure 15 shows some of these to identify the shear failure occurred in the pullout samples. SEM images clearly display the nature of failure of the pullout sample after testing. The 'dent' on the blob shows the pullout force being exerted on the blob, in effect shearing off the fiber bundle from the blob interface (Figures a and b). Some of the load in the initial stage is exerted in creation of this matrix deformation, which is visible in the load data as a slight horizontal 
curve in the initial stage. It can be observed from the images that the major failure caused is not to the matrix or to the fiber but in the interface.

Figures $\mathrm{c}$ and $\mathrm{d}$ are of the same blob sample with increased magnification. The blob-fiber interface has clearly sheared off. This can be observed in the form of damage to the blob and the fiber bundle spreading out, i.e., interface being sheared. Matrix damage, fiber pullout, and fiber failure are shown in Fig. 15(d)

There is no visible matrix failure; thus, the crack is expected to initiate at the interface. Figure 15(e) is one of the focused images of the interface. Interface damage, fiber surface damage, etc. can be visualized from Figure 15(e). When the pullout test was performed, a few samples failed by tension before shear failure. In some cases, the blob was not attached to the fiber bundle in a satisfactory way, which caused abnormally low peak pullout force for that sample, and such samples were eliminated from calculations.

\section{CONCLUSION}

Multiple fiber pull-out test is a reliable statistically averaged method of interface analysis in polymer fiber reinforced composites. It can be observed from this study that this method can be developed as a better tool to evaluate the interface properties of polymer composite materials. Interface adhesion is the important parameter in overall performance of composite materials. Influence of contact length and blob length on different interfacial properties was studied. Variation of some parameters in double bundle from the expected results compared to triple bundle could be due to the uniformity and close packing of the bundles of triple fibers when they are processed. Much higher volume fractions of the bundles can also be tested in the similar fashion as an extension of this study. It will be challenging to get the control over the various parameters like blob shape and size in this method of multiple fiber pull-out test. An optimum bundle size, blob shape, contact angle, and contact length are to be decided and maintained in order to get reduced dispersion in the results.

\section{ACKNOWLEDGMENT}

The authors acknowledge the support throughout this work by the School of Mechanical engineering for their.

\section{REFERENCES}

Aktas, M, Atas, C \& Icten, BM. 2009. An experimental investigation on impact response of unidirectional composite laminates. Composite Structures, 87(4): 307-13.

Bárány, T., Izer, A. and Karger-Kocsis, J. (2009). 'Impact resistance of all-polypropylene composites composed of alpha and beta modifications', Polymer Testing, 28(2), pp. 176-182. doi: 10.1016/j.polymertesting.2008.11.011.

Chandran, M.S. et al. (2018). 'Fabrication and mechanical analysis of jute-sisal hybrid composite', ARPN Journal of Engineering and Applied Sciences, 13(5).

Chandran, M.S. and Padmanabhan, K. (2020). 'Materials Today : Proceedings A novel correlative formulation of interfacial, quasi-static and dynamic behavior of polyamide self reinforced polymer composites', (xxxx).

Désarmot, G. and Favre, J.P. (1991). 'Advances in pull-out testing and data analysis', Composites Science and Technology, 42(1-3), pp. 151-187. doi: 10.1016/0266-3538(91)90016-I.

Gao, C. et al. (2012). 'Development of self-reinforced polymer composites', Progress in Polymer Science. Elsevier Ltd, 37(6), pp. 767-780. doi: 10.1016/j.progpolymsci.2011.09.005.

Jiang, K.R. and Penn, L.S. (1992). 'Improved analysis and experimental evaluation of the single filament pull-out test', Composites Science and Technology, 45(2), pp. 89-103. doi: 10.1016/0266-3538(92)90031-W.

Krishnan, P. (2017). 'A novel microbond bundle pullout technique to evaluate the interfacial properties of fibre-reinforced plastic composites', Bulletin of Materials Science, 40(4), pp. 737-744. doi: 10.1007/s12034-017-1415-z.

Miller, B., Gaur, U. and Hirt, D.E. (1991). 'Measurement and mechanical aspects of the microbond pull-out technique for obtaining fiber/resin interfacial shear strength', Composites Science and Technology, 42(1-3), pp. 207-219. doi: 10.1016/0266- 
3538(91)90018-K.

Minnicino, M.A. and Santare, M.H. (2012). 'Modeling the progressive damage of the microdroplet test using contact surfaces with cohesive behavior', Composites Science and Technology, 72(16), pp. 2024-2031. doi: 10.1016/j.compscitech.2012.09.009.

Morgan, L.M. et al. (2009). 'Self reinforced polymer composites : coming of age', 17th International Conference on Composite Materials, p. ID12.15. Available at: http://www.iccm-central.org/Proceedings/ICCM17proceedings/Themes/Materials/ THERMOPLA MATRIX COMP/INT - THERMOPL MAT COMP/ID12.15 Morgan.pdf.

Raman, S. et al. (2013). 'Fabrication and testing of jute Epoxy Biocomposite', International Journal of Applied Engineering Research, 8(19 SPEC.ISSUE).

Raman, S., Salil Kumar, C.S. and Sharan Chandran, M. (2014). 'Fabrication, testing and analysis of braided and short fibre reinforced jute epoxy bio-composite', International Journal of ChemTech Research, 6(3).

Sharan Chandran, M. et al. (2014). 'Processing and mechanical characterization of self reinforced polymer composite systems', International Journal of ChemTech Research, 6 (6 SPEC. ISS.).

Wang, H.H. et al. (2013). 'An improved microbond test method for determination of the interfacial shear strength between carbon fibers and epoxy resin', Polymer Testing. Elsevier Ltd, 32(8), pp. 1460-1465. doi: 10.1016/j.polymertesting.2013.09.017.

Zhandarov, S. and Mäder, E. (2016). 'Determining the interfacial toughness from force-displacement curves in the pull-out and microbond tests using the alternative method', International Journal of Adhesion and Adhesives. Elsevier, 65, pp. 11-18. doi: 10.1016/j.ijadhadh.2015.10.020.

Zhi, C., Long, H. and Miao, M. (2018). 'Microbond testing and finite element simulation of fibre-microballoon-epoxy ternary composites’, Polymer Testing. Elsevier, 65(December 2017), pp. 450-458. doi: 10.1016/j.polymertesting.2017.12.029. 